\title{
Research on the Reliability Allocation Method for a Production System Based on Availability
}

\author{
Yankun Wang $\mathbb{D}^{1}{ }^{1}$ Binbin $\mathrm{Xu},{ }^{2}$ Tianqi $\mathrm{Ma},{ }^{1}$ and Ziyue Wang ${ }^{1}{ }^{1}$ \\ ${ }^{1}$ School of Mechanical and Aerospace Engineering, Jilin University, Changchun 130025, China \\ ${ }^{2}$ Sino-German College of Intelligent Manufacturing, Shenzhen Technology University, Shenzhen 518118, China \\ Correspondence should be addressed to Ziyue Wang; ziyue18@mails.jlu.edu.cn
}

Received 29 September 2019; Revised 13 December 2019; Accepted 3 January 2020; Published 22 January 2020

Academic Editor: Elena Zaitseva

Copyright (C) 2020 Yankun Wang et al. This is an open access article distributed under the Creative Commons Attribution License, which permits unrestricted use, distribution, and reproduction in any medium, provided the original work is properly cited.

This paper involves a production system, which is composed of units (workstations) and buffers. The buffer is used to store semifinished and finished products in the production process, to reduce the impacts of bad equipment in the production system on the entire system performance. Considering the characteristics of the large number of components and the state of the buffers in the production system, this paper considers the influences of buffer states on upstream and downstream units. When using the availability as the allocation index and combining it with Markov theory, the production unit (workstation) and upstream and downstream buffers are regarded as an equivalent unit (workstation) with multiple output states. We establish the relationship between the availability of each equivalent unit (workstation) and the production system availability and determine a scaling factor for the availability of the equivalent unit to account for the system availability. The expected availability goal of the production system is allocated to each equivalent unit (workstation) by the scaling factor; then, the availability of each equivalent unit (workstation) is assigned to each unit. Finally, the Plant Simulation software is used to simulate and analyze the production system to verify the correctness of the allocation method and realize the reliability allocation from a complex production system to a unit.

\section{Introduction}

A production system is a complex repairable system with buffers. The buffer is used to store semifinished and finished products to reduce the influences of bad equipment in the production system on the entire system performance and ensure continuous production, which is related to the overall efficiency and success of the enterprise. Reliability allocation refers to assigning the reliability index specified in the design specification to each product component in the product design stage [1]. For the production system, the reliability allocation is mainly oriented to the initial design stage. According to the purpose of the entire system, the relevant reliability index is set and allocated to each relevant unit. Reliability allocation is one of the most critical tasks in system reliability design. Whether the allocation result is reasonable directly affects the realization of the reliability index and the design cycle and life cycle cost of the product, and the result directly influences a product's quality and the robustness of the system. The reliability allocation of a production system must consider the influences of the buffers, the polymorphism of the state of buffers, and the large number of components of the production system, so its reliability allocation algorithm is complex and difficult to use [2-5].

Various reliability allocation methods have been widely discussed and developed over the last several decades. In response to the reliability allocation problem of production systems, Jilin University CNC Equipment Reliability Technology Innovation Research Team conducted research on a car crankshaft production line and an engine block production line $[6,7]$, established a fuzzy comprehensive evaluation model for production line reliability allocation [8], and proposed a seminumerical simulation method for the availability evaluation of discrete production lines [9]. Based on the availability of equipment, the availability of 
production lines, and the cost of the three indicators, they proposed a production line reliability assessment method [10]. Availability is an important indicator to evaluate and analyze the reliability of production lines. The research methods for production line reliability assessment include the Petri net model [11], the fuzzy Bayesian method [12], the Markov model [13, 14], and the semi-Markov model $[15,16]$. Heungseob and Pansoo [14] developed a model for nonrepairable systems with heterogeneous components with phase-type time-to-failure distributions by using a structured continuous-time Markov chain (CTMC). Loganathan et al. [15] used the semi-Markov model to evaluate the availability of a manufacturing system that considered variable failure rates or maintenance rates.

At present, there are many studies on reliability allocation methods, but few of them are applicable to production systems. The reliability allocation of a production system usually allocates the system equipment levels and does not consider the buffer area. The buffer area can improve the reliability index of the production system. Hu and Meerkov [17] have shown that the equipment obeys the Bernoulli reliability model and proposed an analysis method to select the lean buffer for serial production lines. Demir et al. [18] used the decomposition method to obtain the throughput evaluation model for asynchronous production lines and used an adaptive tabu search algorithm to determine the buffer capacity of the production line.

A production system is a multistate system, which often consists of multiple processing units, and there may be parallel units. In addition, there is a buffer between processing units. These factors make the working state of the production system appear polymorphic. For a multistate system, the Markov model [19], a Bayesian network $[20,21]$, a fuzzy mathematical method $[8,22]$, and other methods are currently used. The Markov model is used to construct the reliability model by dynamically describing the system state and the transition of the state from two aspects of the system. This method can comprehensively analyze the reliability of the system. However, when the system levels increase, the number of system states sharply increases, and the analysis results exponentially increase. The algorithm is complex and difficult to solve. The Bayesian network can describe the polymorphism of the system and the uncertainty of the logical relationship between events, perform two-way reasoning, and find the source of system failure in the reliability analysis. These advantages make the Bayesian network to be widely used in system reliability analysis. However, for complex multistate systems, when the number of variables is large or the range of the variables is large, the scale and complexity of the local conditional probability table will increase as an exponential function, which makes it difficult to learn conditional probability parameters and affects the practicality of the entire network model. The fuzzy mathematical method is suitable for reliability allocation under the condition of uncertain parameters. There are many uncertain fuzzy factors to be faced in the allocation process. It is precise to use the fuzzy mathematical method to address these types of inaccurate parameters, which will have better results.
However, the research on fuzzy reliability allocation is mostly limited to the system reliability allocation problem with a simple structure, and many factors must be considered in reliability allocation. The quantitative expression of each factor generally requires the participation of experts, and the results given by experts are often highly subjective, which increases the fuzziness of the reliability allocation.

In this paper, buffer areas are introduced into the reliability allocation of the production system. By analyzing the calculation process of the availability of the production system, the quantitative relationship between the availability of equivalent units (workstations) including the buffers and units and the availability of the system is calculated. The concept of the scale factor is proposed. A method to allocate the system availability according to the availability factor of each equivalent unit to the system availability ratio is proposed, and the expected availability target of the production system is allocated to each constituent unit. Finally, the production system is simulated and analyzed by the Plant Simulation software to verify the correctness of the allocation method. The method realizes the reliability allocation from complex production systems to units, which is easy to implement in engineering and has strong feasibility. It can also solve the reliability allocation problem of the multistage production system and provide a basis for the design, transformation, and upgrade of the production system.

According to the composition of the repairable system, this paper divides the system into two categories: an unbuffered system of rigid connections between every two constituent unit and a nonrigid connection system that considers a buffer between constituent units. The remainder of this paper is organized as follows: Section 2 establishes the mathematical relationship between the unbuffered system availability and the unit availability of the series and seriesparallel systems, respectively. Section 3 first establishes the mathematical relationship between the buffer system availability and the unit availability of the series and seriesparallel hybrid systems. Then, a method of buffer inventory capacity allocation is introduced. Section 4 establishes the availability allocation method for the unbuffered system and buffer system. Section 5 introduces the three stages of the Plant Simulation software in the simulation process. In Section 6, a case is presented and simulated by the software to illustrate the rationality of the proposed allocation method. Section 7 concludes this paper.

\section{Establishing a Mathematical Relationship between Unbuffered System Availability and Unit Availability}

2.1. Establishing a Mathematical Relationship between Unbuffered Series System Availability and Unit Availability. The system $\mathrm{S}$ consists of $i(i=1,2, \ldots, n)$ series units; each unit is recorded as $m_{i}$. The reliability block diagram is shown in Figure 1.

Assuming that the failure rate and maintenance rate of $m_{i}$ are $\lambda_{i}$ and $\mu_{i}$, and the lifetime and maintenance time obey 


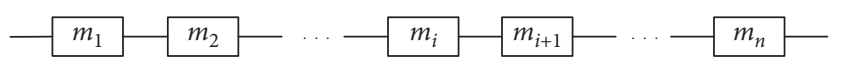

FIGURE 1: Reliability block diagram of an unbuffered series system.

the exponential distributions with parameters $\lambda_{i}$ and $\mu_{i}$, the steady-state availability of the series unit is

$$
a_{i}=\frac{\mu_{i}}{\lambda_{i}+\mu_{i}} .
$$

The steady-state availability of the system is

$$
A=\frac{1}{1+\sum_{i=1}^{n} \lambda_{i} / \mu_{i}} .
$$

2.2. Establishing a Mathematical Relationship between Unbuffered Parallel-Series System Availability and Unit Availability. The parallel-series system consists of parallel units that are put in series. For the parallel-series system with rigid connections between units, in this case, the production system must be simplified to a standard serial system, and the parallel units are converted into an equivalent unit, which facilitates the next analysis and improvement.

In this paper, two identical units $m_{i, L}$ and $m_{i, R}$ are arranged in parallel on the layout as an example, and the subsystem containing parallel units is replaced by the equivalent unit $m_{i}^{\text {par }}$. The reliability block diagram is shown in Figure 2.

Assuming that the failure rate and repair rate of $m_{i, L}$ and $m_{i, R}$ are $\lambda_{i}$ and $\mu_{i}$, respectively, the steady-state availability of the equivalent unit $m_{i}^{\mathrm{par}}$ is

$$
a_{i}^{\text {par }}=\frac{\mu_{i}^{2}+2 \lambda_{i} \mu_{i}}{\mu_{i}^{2}+2 \lambda_{i} \mu_{i}+2 \lambda_{i}^{2}} .
$$

Expansion of equation (2) yields

$$
A=\frac{1}{\left(1+\left(\lambda_{1} / \mu_{1}\right)\right)+\left(1+\left(\lambda_{2} / \mu_{2}\right)\right)+\cdots+\left(1+\left(\lambda_{n} / \mu_{n}\right)\right)-(n-1)},
$$

where $1+\left(\lambda_{i} / \mu_{i}\right)=\left(1 / a_{i}\right)$; the relationship between system availability and unit availability can be expressed as

$$
A=\frac{1}{\left(1 / a_{1}\right)+\left(1 / a_{2}\right)+\cdots+\left(1 / a_{n}\right)-(n-1)} .
$$

We further formulate equation (5) as

$$
\left(\frac{1}{a_{1}}-1\right)+\left(\frac{1}{a_{2}}-1\right)+\left(\frac{1}{a_{3}}-1\right)+\cdots+\left(\frac{1}{a_{n}}-1\right)=\left(\frac{1}{A}-1\right) \text {. }
$$

Dividing both sides of equation $(6)$ by $((1 / A)-1)$ yields the following formula:

$$
c_{1}+c_{2}+c_{3}+\cdots+c_{n}=1
$$

where $c_{i}=\left(\left(1 / a_{i}\right)-1\right) /((1 / A)-1)$, and equation (7) expresses the relationship between unit availability and system availability.

\section{Establishing a Mathematical Relationship between Buffer System Availability and Unit Availability}

3.1. Establishing a Mathematical Relationship between Buffer Series System Availability and Unit Availability. The series system connects units $m_{i}(i=1,2, \ldots, n)$ in series and transfers the workpiece to the next-level unit $m_{i+1}$ through the buffer $B_{i}$. The reliability block diagram is shown in Figure 3.

Taking $m_{i}$ as an example, productivity refers to the number of products that $m_{i}$ can produce per unit time; starvation refers to the forced waiting caused by the lack of workpiece to provide to $m_{i}$ after $m_{i}$ processes, which releases a workpiece, and the capacity $k_{i-1}$ of the upstream buffer $B_{i-1}$ is zero (empty); and blocking refers to the forced waiting caused by the capacity $k_{i}$ of the downstream buffer $B_{i}$ being $n$ (full), which makes the workpiece unable to put into the buffer.

We assume that the lifetime and maintenance time of $m_{i}$ obey the exponential distributions with parameters $\lambda_{i}$ and $\mu_{i}$, the first-level unit is not starved, and the last buffer is not blocked. When the buffer capacity $k_{i}$ of the buffer $B_{i}$ is $n$ (full), the previous unit $m_{i}$ is down and the next-level unit $m_{i+1}$ continues working. When the buffer capacity $k_{i}$ of the buffer $B_{i}$ is zero (empty), the next-level unit $m_{i+1}$ is down. Increasing the buffer can effectively improve the unit availability. When the unit fails, the spare parts in the buffer can maintain production for a period of time and strive for maintenance time. References [5, 23] have analyzed and deduced the state of the buffer in detail.

Taking $B_{i}$ as an example, inventory-free refers to the capacity of $B_{i}$ being $k_{i}=0$; inventory refers to the capacity of $B_{i}$ being $k_{i}>0$; vacancy-free refers to the capacity of $B_{i}$ being $k_{i}=n$; and vacancy refers to the capacity of $B_{i}$ being $k_{i}<n$. Assume that the probability of inventory-free is $P_{0 i}$, the probability of inventory is $P_{\overline{0 i}}$, the probability of vacancyfree is $P_{k_{i}}$, and the probability of vacancy is $P_{\overline{k_{i}}}$, then the calculation equation for the buffer availability is as follows:

$$
A_{B_{i}}=P_{\overline{0(i-1)}} P_{\overline{k_{i}}}
$$

where

$$
\begin{aligned}
& P_{\overline{0 i}}=\frac{\rho_{i} \cdot\left(1-\rho_{i}^{k_{i}}\right)}{\left(1-\rho_{i}^{k_{i}+1}\right)}, \\
& P_{\overline{k_{i}}}=\frac{\left(1-\rho_{i}^{k_{i}}\right)}{\left(1-\rho_{i}^{k_{i}+1}\right)}, \\
& \rho_{i}=\frac{\omega_{i}}{\omega_{i+1}} .
\end{aligned}
$$

Each unit and its upstream and downstream buffers are equivalent to the unit $m_{i}^{\prime}$ with multiple output states. We establish the state transition probability equation of the $i$-th equivalent unit and obtain the availability of $m_{i}^{\prime}[23]$ : 


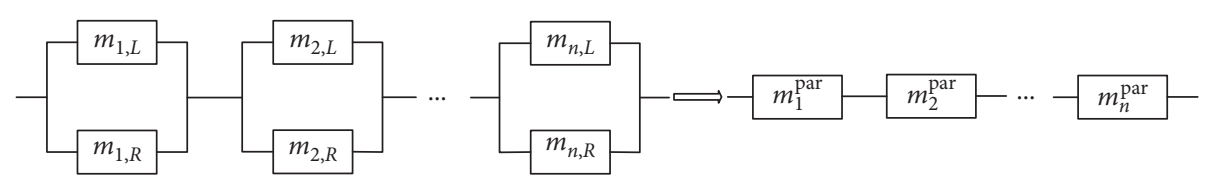

FiguRE 2: Reliability block diagram of an unbuffered parallel-series system.

$$
\begin{aligned}
& -m_{1}-\left(B_{1}-m_{2}-\left(B_{2}-\cdots-m_{i}-B_{i}-m_{i+1}-\cdots-m_{n}\right.\right. \\
& A_{i}=\frac{\mu_{i} A_{B i}}{\left(\mu_{i}+\lambda_{i} A_{B i}\right)}=\frac{\mu_{i} P_{\overline{0(i-1)}} P_{\overline{k i}}}{\left(\mu_{i}+\lambda_{i} P_{\overline{0(i-1)}} P_{\overline{k i}}\right)}=\frac{\mu_{i}\left(\rho_{i-1} \cdot\left(1-\rho_{i-1}^{k(i-1)}\right) /\left(1-\rho_{i-1}^{k(i-1)+1}\right)\right)}{\left[\mu_{i}+\lambda_{i}\left(\rho_{i-1} \cdot\left(1-\rho_{i-1}^{k(i-1)}\right) /\left(1-\rho_{i-1}^{k(i-1)+1}\right)\right)\left(\left(1-\rho_{i}^{k i}\right) /\left(1-\rho_{i}^{k i+1}\right)\right)\right]} .
\end{aligned}
$$

According to equation (12), the availability of the equivalent unit is directly related to the failure rate, maintenance rate, productivity, and buffer inventory capacity of the unit. If the availability index of each unit is determined, equation (12) can be used to guide the selection of production equipment and determine the buffer inventory capacity.

\subsection{Establishing a Mathematical Relationship between Buffer} Parallel-Series Hybrid System Availability and Unit Availability. The buffer parallel-series hybrid system consists of two or more units arranged in parallel on the layout to form a workstation $M_{i}(i=1,2, \ldots, n)$, and workstations $M_{i}$ and $M_{i+1}$ are connected in series through the buffer $B_{i}(i=1,2, \ldots, n-1)$. In this paper, the parallel distribution of two units $m_{i, L}$ and $m_{i, R}$ in the layout is taken as an example, and the reliability block diagram is shown in Figure 4.

When each workstation $M_{i}$ in the parallel-series hybrid system is composed of two units in parallel, there are three states of the workstation: (1) both units are normal and $M_{i}$ works normally; (2) one unit fails and $M_{i}$ reduces production; and (3) both units fail and $M_{i}$ fails. Combined with the state of the upstream and downstream buffers, $M_{i}$ has nine working states:

(1) The probability of $M_{i}$ working normally is $P_{a_{i}}^{\prime}=P_{\overline{0(i-1)}} P_{a_{i}} P_{\overline{k_{i}}}$.

(2) $M_{i}$ is trouble-free, and the input shortage causes discontinuation. The probability is $P_{0(i-1)} P_{a_{i}} P_{\overline{k_{i}}}$.

(3) $M_{i}$ is trouble-free, and the output blocking causes discontinuation. The probability is $P_{\overline{0(i-1)}} P_{a_{i}} P_{k_{i}}$.

(4) $M_{i}$ is trouble-free; the input shortage and output blocking cause discontinuation. The probability is $P_{0(i-1)} P_{a_{i}} P_{k_{i}}$.

(5) The probability of $M_{i}$ reducing production is $P_{c_{i}}^{\prime}=P_{\overline{0(i-1)}} P_{c_{i}} P_{\overline{k_{i}}}$.

(6) $M_{i}$ reduces production, and the input shortage causes discontinuation. The probability is $P_{0(i-1)} P_{c_{i}} P_{\bar{k}_{i}}$.

(7) $M_{i}$ reduces production, and the output blocking causes discontinuation. The probability is $P \overline{0(i-1)} P_{c_{i}} P_{k_{i}}$.
(8) $M_{i}$ reduces production; the input shortage and output blocking cause discontinuation. The probability is $P_{0(i-1)} P_{c_{i}} P_{k_{i}}$.

(9) The probability of $M_{i}$ stopping production due to malfunction is $P_{b_{i}}$.

By adding the above probabilities,

$$
\begin{aligned}
& P_{a_{i}}\left(P_{\overline{0(i-1)}} P_{\overline{k_{i}}}+P_{0(i-1)} P_{\overline{k_{i}}}+P_{\overline{0(i-1)}} P_{k_{i}}+P_{0(i-1)} P_{k_{i}}\right) \\
& \quad+P_{c_{i}}\left(P_{\overline{0(i-1)}} P_{\bar{k}_{i}}+P_{0(i-1)} P_{\bar{k}_{i}}+P_{\overline{0(i-1)}} P_{k_{i}}+P_{0(i-1)} P_{k_{i}}\right) \\
& \quad+P_{b_{i}}=1 .
\end{aligned}
$$

We can prove that the four probabilities in brackets of equation (13) add up to 1 , so that

$$
P_{a_{i}}+P_{c_{i}}+P_{b_{i}}=1 \text {. }
$$

The state transition probability equation is

$$
\left\{\begin{array}{l}
\dot{P}_{a_{i}}^{\prime}=-2 \lambda_{i} P_{a_{i}}^{\prime}+\mu_{i} P_{c_{i}}^{\prime}, \\
\dot{P}_{c_{i}}^{\prime}=2 \lambda_{i} P_{a_{i}}^{\prime}-\left(\lambda_{i}+\mu_{i}\right) P_{c_{i}}^{\prime}+\mu_{i} P_{b_{i}}, \\
\dot{P}_{b_{i}}=\lambda_{i} P_{c_{i}}^{\prime}-\mu_{i} P_{b_{i}} .
\end{array}\right.
$$

We solve (15) and obtain

$$
\begin{aligned}
& P_{c_{i}}^{\prime}=\frac{2 \lambda_{i} \mu_{i} A_{B_{i}}}{\mu_{i}^{2}+2 \lambda_{i}^{2} A_{B_{i}}+2 \lambda_{i} \mu_{i}}, \\
& P_{a_{i}}^{\prime}=\frac{\mu_{i}^{2} A_{B_{i}}}{\mu_{i}^{2}+2 \lambda_{i}^{2} A_{B_{i}}+2 \lambda_{i} \mu_{i}}, \\
& P_{b_{i}}=\frac{2 \lambda_{i}^{2} A_{B_{i}}}{\mu_{i}^{2}+2 \lambda_{i}^{2} A_{B_{i}}+2 \lambda_{i} \mu_{i}} .
\end{aligned}
$$

Considering the influence of the buffer availability on the system availability, the buffer available state is that its upstream buffer is not starved and its downstream buffer is not blocked. Combining the two states with the workstation as an equivalent workstation $M_{i}^{\prime}$ for analysis, we obtain the steady-state availability of $M_{i}^{\prime}$ : 


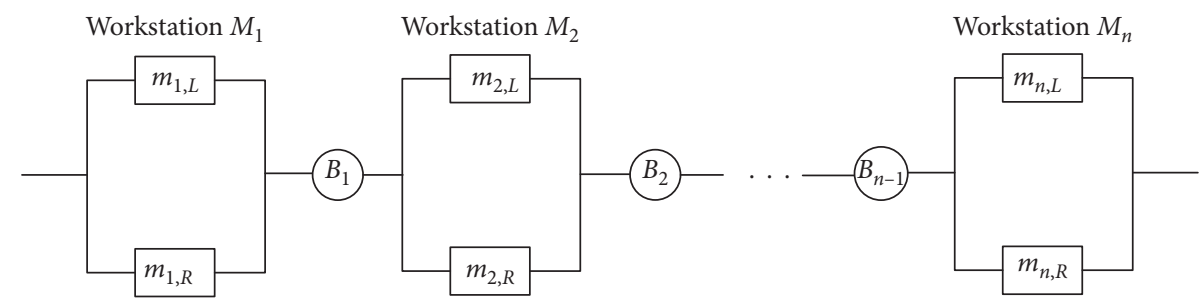

FIgURE 4: Reliability block diagram of a buffer parallel-series hybrid system.

$$
A_{i}^{\prime}=\frac{\left(2 \lambda_{i} \mu_{i}+\mu_{i}^{2}\right) A_{B_{i}}}{\mu_{i}^{2}+2 \lambda_{i}^{2} A_{B_{i}}+2 \lambda_{i} \mu_{i}} .
$$

The steady-state unavailability of the system is equal to the product of the unavailability of each equivalent workstation, i.e.,

$$
\bar{A}=\prod_{i=1}^{n}\left(1-A_{i}^{\prime}\right) .
$$

Thus, the steady-state availability of the system is

$$
A=1-\bar{A}=1-\prod_{i=1}^{n}\left(1-A_{i}^{\prime}\right) \text {. }
$$

Formula (19) is expanded and transformed into

$$
\begin{aligned}
& \left(1-A_{1}^{\prime}\right)\left(1-A_{2}^{\prime}\right)\left(1-A_{3}^{\prime}\right) \cdots\left(1-A_{n-2}^{\prime}\right)\left(1-A_{n-1}^{\prime}\right)\left(1-A_{n}^{\prime}\right) \\
& \quad=1-A .
\end{aligned}
$$

Simultaneously, taking the logarithm of both sides yields

$$
\begin{aligned}
& \ln \left(1-A_{1}^{\prime}\right)+\ln \left(1-A_{2}^{\prime}\right)+\ln \left(1-A_{3}^{\prime}\right)+\cdots \\
& \quad+\ln \left(1-A_{n-2}^{\prime}\right)+\ln \left(1-A_{n-1}^{\prime}\right)+\ln \left(1-A_{n}^{\prime}\right)=\ln (1-A) .
\end{aligned}
$$

Dividing both sides of equation $(21)$ by $\ln (1-A)$ yields the following formula:

$$
c_{1}^{\prime}+c_{2}^{\prime}+c_{3}^{\prime}+\cdots+c_{n-2}^{\prime}+c_{n-1}^{\prime}+c_{n}^{\prime}=1,
$$

where $c_{i}^{\prime}=\left(\ln \left(1-A_{i}^{\prime}\right) / \ln (1-A)\right)$.

Increasing the buffer improves the workstation availability. In this case, the equivalent workstation availability after combining the upstream and downstream buffers will be higher than the availability of the unit itself. Thus, the series-parallel hybrid system can be simplified to a series system that consists of equivalent workstations that contain buffers.

\subsection{Buffer Inventory Capacity Allocation Method.} Figure 5 is a structural diagram of a serial two-level production system composed of workstations $M_{i}$ and $M_{i+1}$ and the buffer $B_{i}$.

Assume that the buffer is completely reliable during the planning period $T$. The failure rate and maintenance rate are $\lambda_{B}$ and $\mu_{B}$, respectively. $B_{i}$ is separately treated as a unit, and the steady-state availability of $B_{i}$ is

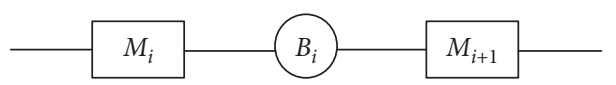

FIGURE 5: Structural diagram of a serial two-level production system.

$$
A_{B}=\frac{\mu_{B}}{\mu_{B}+\lambda_{B}}
$$

Considering the occasional malfunction of $B_{i}$ in actual production, the correction factor $\varepsilon$ is introduced in this paper, and $\varepsilon=\left(1 / A_{B}\right)$. During the planning period time $T$, to ensure the continuous production of the system, the minimum buffer inventory capacity $k_{i, \min }$ is [24]

$$
\left\{\begin{array}{l}
k_{i, \min }=\varepsilon \cdot \max \left\{k_{i, 1}, k_{i, 2}\right\}, \\
k_{i, 1}=k_{u}\left[\frac{1}{\mu_{B}}\left(1-e^{-\mu_{B} T}\right)-T e^{-\mu_{B} T}\right]+1, \\
k_{i, 2}=2 k_{u}\left[\frac{1}{\mu_{B}}\left(1-e^{-\mu_{B} T}\right)-T e^{-\mu_{B} T}\right] \\
-\left(k_{p}-k_{u}\right)\left[\frac{1}{\lambda_{B}}\left(1-e^{-\lambda_{B} T}\right)-T e^{-\lambda_{B} T}\right]+1 .
\end{array}\right.
$$

\section{Availability Allocation Method}

4.1. Proportional Allocation Method. The proportional allocation method is based on the failure rate of each unit in the original system, and the failure rate is proportionally distributed to each unit of the new system according to the reliability prediction of the new system. The mathematical expression is [25]

$$
\lambda_{\text {in }}=\frac{\lambda_{\text {io }}}{\lambda_{s o}} \lambda_{s n}
$$

where $\lambda_{s n}$ is the failure rate of the new system; $\lambda_{i n}$ is the failure rate allocated to the unit $i$ in the new system; $\lambda_{s o}$ is the failure rate of the old system; and $\lambda_{i o}$ is the failure rate of the unit $i$ in the old system.

Similarly, this paper uses the system availability as the allocation index, which is proportionally allocated to each unit (workstation) according to the scale factor to determine the unit (workstation) availability index value. 
4.2. Availability Allocation Method for an Unbuffered System. Assume that the production system availability is $A$ and the unit availability is $a_{i}$. A and $a_{i}$ satisfy formula (6), and the influence factor is $c_{i}$. If the system target availability is $A_{m}$ and the availability assigned to each unit is $A_{i m}$, then

$$
\left(\frac{1}{A_{\text {im }}}-1\right)=c_{i}\left(\frac{1}{A_{m}}-1\right) \text {. }
$$

The unit availability $A_{i m}$ after sorting is

$$
A_{\text {im }}=\frac{1}{\left[c_{i}\left(\left(1 / A_{m}\right)-1\right)+1\right]} \text {. }
$$

4.3. Availability Allocation Method for a Buffer System. If $k_{i}<n$ and $k_{i+1}<n$, the system target availability is $A_{m}^{\prime}$, the availability assigned to each workstation is $A_{c_{i}}^{\prime}$, and the scale factor is $c_{i}^{\prime}$; then,

$$
\begin{aligned}
c_{i}^{\prime} & =\frac{\ln \left(1-A_{c_{i}}^{\prime}\right)}{\ln \left(1-A_{m}^{\prime}\right)}, \\
A_{c_{i}}^{\prime} & =1-e^{c_{i}^{\prime} \ln \left(1-A_{m}^{\prime}\right)} .
\end{aligned}
$$

$A_{c_{i}}^{\prime}$ is assigned to each unit by an equal reliability allocation method of the parallel system. Assume that the availability assigned to each unit is $A_{m_{i}}^{\prime}$, then the allocation method formula is

$$
\begin{aligned}
& \overline{A_{m_{i}}^{\prime}}=\sqrt{\overline{A_{c_{i}}^{\prime}}}=\sqrt{1-A_{c_{i}}^{\prime}}=\sqrt{\overline{e^{c_{i}^{\prime}} \ln \left(1-A_{m}^{\prime}\right)}}, \\
& A_{m_{i}}^{\prime}=1-\sqrt{\overline{A_{m_{i}^{\prime}}^{\prime}}}=1-\sqrt{\overline{e^{c_{i}^{\prime}} \ln \left(1-A_{m}^{\prime}\right)}} .
\end{aligned}
$$

\section{Simulation Analysis for the Production System}

The simulation establishes a model for the system and uses the model instead of the real system to perform various experiments to study the performance. In this paper, the Plant Simulation software is used as the simulation platform. After obtaining the availability allocation results, the system is simulated and analyzed to verify the correctness of the results.

The simulation of the production system corresponds to three stages:

(1) Establishing a simulation model: using the "logistics," the production equipment and buffer can be obtained, and the basic system framework model can be established. For each unit, the processing capacity and availability are input; for each buffer, the buffer capacity, type, and availability are set.

(2) Performing a simulation experiment: using the event control unit, the simulation time is set to 30 days, 90 days, 180 days, 360 days, and 720 days. This time is set to absolute time, which is convenient for observing and recording simulation events. The corresponding time jump speed is 10,000 times the real-time value, and the remaining options are default values. The parameters of relevant production units are set, and the software to simulate the entire line is run.

(3) Analyzing the simulation data: after the simulation test is completed, the intrinsic availability, total throughput, hourly throughput, and daily throughput related data are collated and compared with the given expected availability. According to the comparison result, it is determined whether the unit reliability index can achieve the expected goal and whether the reliability allocation method is correct.

\section{Examples}

Assume that a buffer series-parallel hybrid production system consists of a 4-level workstation. Each level workstation consists of two identical processing units connected in parallel. The failure rate, maintenance rate, and productivity of each unit are $\lambda_{1} \lambda_{2} \lambda_{3} \lambda_{4}, \mu_{1} \mu_{2} \mu_{3} \mu_{4}$, and $\omega_{1} \omega_{2} \omega_{3} \omega_{4}$, respectively, which are listed in Table 1 .

Assume that the failure rate $\lambda_{B i}$ and maintenance rate $\mu_{B i}$ of each buffer are 0.002 and 0.06 , the planning period time $T$ is 10 minutes, and $k_{p}$ and $k_{u}$ are 1 minute per piece. Substituting parameters into equations (23) and (24), we obtain $A_{B 1}=A_{B 2}=A_{B 3}=0.9677$ and $k_{1}=k_{2}=k_{3}=5$.

It is also assumed that the first-level workstation is not starved and the last-level workstation is not blocked. Let $P_{\overline{00}}=1$ and $P_{\overline{4}}=1$. The buffer availability can be calculated by formulas (8)-(11). The specific values are shown in Table 2.

The steady-state availability of each equivalent workstation can be obtained by using equation (17) and is summarized in Table 3.

By substituting the obtained steady-state availability into (19), the stable availability of the system is $A=1-\left(\prod_{i=1}^{4}\left(1-A_{i}^{\prime}\right)\right)=0.9925$. If the expected availability is increased to 0.9999 , the estimated availability assigned to each workstation is calculated according to equations (28) and (29), and the specific values are shown in Table 4.

The estimated availability of each workstation is redistributed to each unit according to formulas (30) and (31), and the expected unit availability is calculated and shown in Table 5.

After obtaining the estimated availability allocation results of each processing unit, the Plant Simulation software is used to simulate the production system online to determine whether the allocation results are correct and reasonable. Using the "logistics" element in the software element toolbox, the production unit and buffer can be obtained. The simulation model of the production system is shown in Figure 6 .

We input the corresponding parameters for each unit and buffer and use the event control unit to set the simulation time to 30 days, 90 days, 180 days, 360 days, and 720 days, and the corresponding time jump speed is 10,000 times the real-time value. 
TABLE 1: Failure rate, maintenance rate, and productivity of each unit.

\begin{tabular}{lcccccccc}
\hline Parameter & $m_{1, L}$ & $m_{1, R}$ & $m_{2, L}$ & $m_{2, R}$ & $m_{3, L}$ & $m_{3, R}$ & $m_{4, L}$ & $m_{4, R}$ \\
\hline$\lambda_{i}$ & 0.0032 & 0.0032 & 0.0027 & 0.0027 & 0.0026 & 0.0026 & 0.003 \\
$\mu_{i}$ & 0.02 & 0.02 & 0.03 & 0.03 & 0.02 & 0.02 & 0.01 \\
$\omega_{i}$ & 5 & 5 & 4 & 4 & 3.5 & 3.5 & 0.01 \\
\hline
\end{tabular}

TABLE 2: Buffer availability.

\begin{tabular}{ccccc}
\hline$B_{i}$ & $B_{1}$ & $B_{2}$ & $B_{3}$ & $B_{4}$ \\
\hline$A_{B_{i}}$ & 0.7289 & 0.7045 & 0.6745 & 0.8146 \\
\hline
\end{tabular}

TABLE 3: Steady-state availability of each equivalent workstation.

\begin{tabular}{ccccc}
\hline$M_{i}^{\prime}$ & $M_{1}^{\prime}$ & $M_{2}^{\prime}$ & $M_{3}^{\prime}$ & $M_{4}^{\prime}$ \\
\hline$A_{i}^{\prime}$ & 0.7089 & 0.6978 & 0.6625 & 0.7462 \\
\hline
\end{tabular}

TABLE 4: Estimated availability of each workstation.

\begin{tabular}{ccccc}
\hline$M_{i}$ & $M_{1}$ & $M_{2}$ & $M_{3}$ & $M_{4}$ \\
\hline$A_{c i}^{\prime}$ & 0.9022 & 0.8951 & 0.8708 & 0.9245 \\
\hline
\end{tabular}

TABLE 5: Expected availability of each unit.

\begin{tabular}{lcccccccc}
\hline unit & $m_{1, L}$ & $m_{1, R}$ & $m_{2, L}$ & $m_{2, R}$ & $m_{3, L}$ & $m_{3, R}$ & $m_{4, L}$ & $m_{4, R}$ \\
$A_{m_{i}}^{\prime}$ & 0.6873 & 0.6873 & 0.6761 & 0.6761 & 0.6406 & 0.6406 & 0.7253 & 0.7253 \\
\hline
\end{tabular}

\section{$\$$}

Event controller

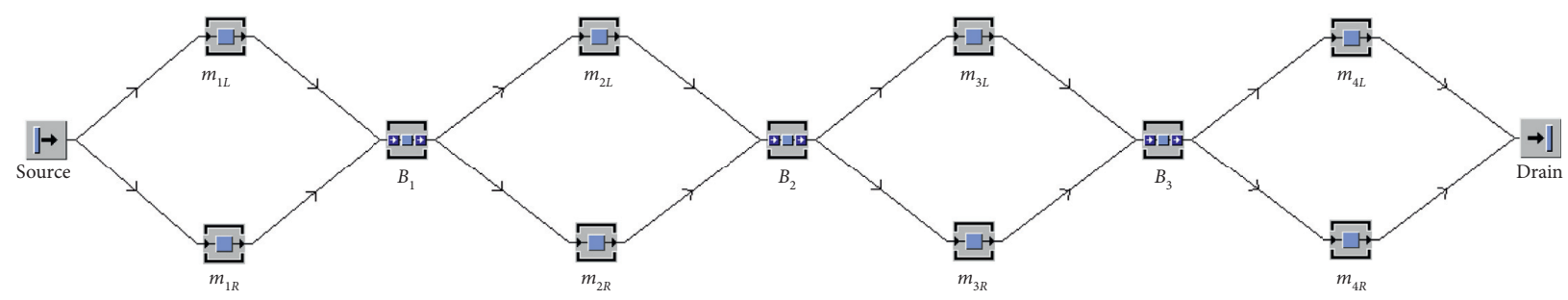

Figure 6: Simulation model of the production system.

(v)

Event controller

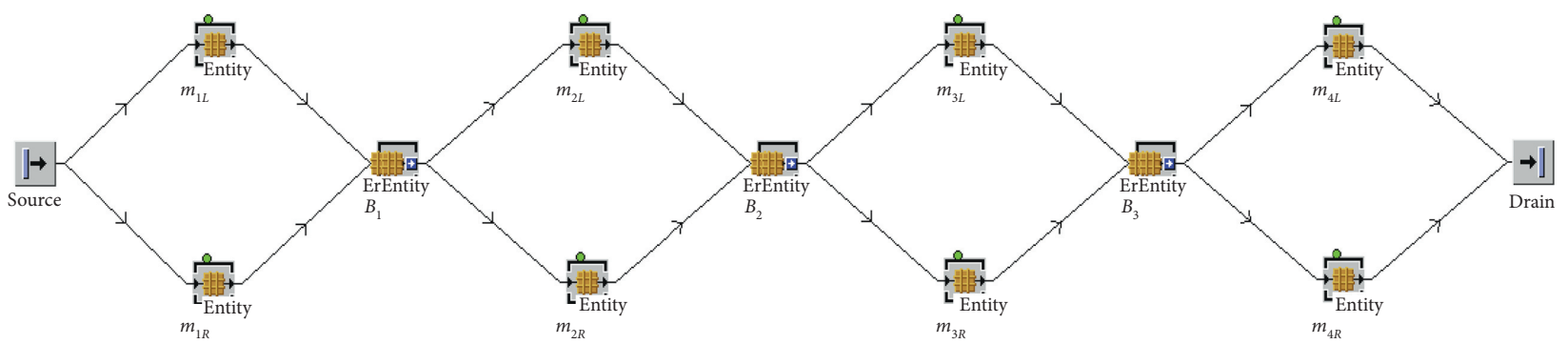

FIGURE 7: Operation simulation model of the production system. 
TABLE 6: Simulation data of the production system.

\begin{tabular}{lcccc}
\hline Time & Intrinsic availability (\%) & Total throughput & Hourly throughput & Daily throughput \\
\hline 30 days & 99.99 & 86386 & 120 & 2880 \\
90 days & 99.99 & 259290 & 121 & 2881 \\
180 days & 99.99 & 518760 & 120 & 2882 \\
360 days & 99.99 & 1037520 & 120 & 2882 \\
720 days & 99.99 & 2075040 & 120 & 2882 \\
\hline
\end{tabular}

We set the parameters of the relevant production units and run the software to simulate the whole line. The operation simulation model is shown in Figure 7.

Then, we complete the simulation test, sort out the relevant data, and obtain the reliability and performance index values. The specific values are shown in Table 6.

The simulation results show that the reliability allocation results obtained in this paper satisfy the requirements of the given expected availability. Therefore, the reliability index allocated to each unit can achieve the desired goal, and the reliability allocation method is correct.

\section{Conclusions}

(1) Aiming at the reliability allocation problem of the production system, this paper proposes a reliability allocation method that is easy to implement in engineering. By constructing the proportional relationship between unit (workstation) availability and system availability, the system availability can be proportionally allocated according to the scale factors. This allocation method is simple and easy to implement, and it can solve the reliability allocation problem of multistage production systems.

(2) The availability allocation results can help guide the selection of production units, determine the number of spare parts in the buffer, comprehensively consider factors such as the failure rate, maintenance rate, inventory capacity, and productivity, and rationally select the reliability index of the processing unit.

(3) Using the Plant Simulation software to simulate and analyze the production system, one can verify the correctness of the allocation method and realize the reliability allocation from the complex production system to the unit.

(4) The reliability allocation process without considering the influence of the buffer can be extended to general series and series-parallel hybrid repairable systems such as CNC machine tools and serve as the basis for component selection or reliability improvement in the product design stage.

\section{Notations}

$\begin{array}{ll}m_{i}: & \text { Unit of a series system }(i=1,2, \ldots, n) \\ \lambda_{i}: & \text { Failure rate of a unit } \\ \mu_{i}: & \text { Maintenance rate of a unit } \\ \omega_{i}: & \text { Productivity of a unit }\end{array}$

$\rho_{i}: \quad$ Productivity ratio of a two-level unit

$a_{i}: \quad$ Steady-state availability of $m_{i}$

A: $\quad$ Steady-state availability of a system

$m_{i, L}$ and Units of a hybrid system $(i=1,2, \ldots, n)$

$m_{i, R}$ :

$m_{i}^{\text {par }}: \quad$ Equivalent unit combining $m_{i, L}$ and $m_{i, R}$

$a_{i}^{\text {par }}$ : $\quad$ Steady-state availability of $m_{i}^{\text {par }}$

$c_{i}$ : $\quad$ Scale factor in an unbuffered hybrid system

$B_{i}: \quad$ Buffers of a system

$k_{i}$ : $\quad$ Inventory capacity of $B_{i}\left(k_{i}=1,2, \ldots, n\right)$

$k_{i, \text { min }}$ : $\quad$ Minimum inventory capacity of $B_{i}$

$\lambda_{B}: \quad \quad$ Failure rate of a buffer

$\mu_{B}$ : $\quad$ Maintenance rate of a buffer

$P_{0 i}$ : $\quad$ Probability of inventory-free

$P_{\overline{0 i}}: \quad$ Probability of inventory

$P_{k_{i}}$ : $\quad$ Probability of vacancy-free

$P_{\bar{k}_{i}}: \quad \quad$ Probability of vacancy

$A_{B_{i}}: \quad$ Buffer availability

$m_{i}^{\prime}: \quad \quad$ Equivalent unit combining $m_{i}, B_{i}$, and $m_{i+1}$ in the buffer series system

$A_{i}: \quad$ Steady-state availability of $m_{i}^{\prime}$

$M_{i}: \quad$ Workstation of a buffer hybrid system $(i=1,2, \ldots, n)$

$P_{a i}: \quad \quad$ Trouble-free probability of $M_{i}$

$P_{b i}$ : $\quad$ Discontinuation probability of $M_{i}$

$P_{c i}: \quad$ Production-reduction probability of $M_{i}$

$M_{i}^{\prime}$ : $\quad$ Equivalent workstation combining $M_{i}, B_{i}$, and $M_{i+1}$ in the buffer hybrid system

$A_{i}^{\prime}$ : $\quad$ Steady-state availability of $M_{i}^{\prime}$

$c_{i}^{\prime}$ : $\quad$ Scale factor in the buffer hybrid system

$k_{p}$ and $k_{u}$ : Output per unit time of $M_{1}$ and $M_{2}$

$A_{m}$ : $\quad$ Target availability of an unbuffered system

$A_{\text {im }}$ : $\quad$ Assigned availability to each unit in the

unbuffered system

$A_{m}^{\prime}$ : $\quad$ Target availability of a buffer system

$A_{c_{i}}^{\prime}$ and Assigned availability and unavailability to the

$\frac{A_{c_{i}}^{\prime}}{A_{c}^{\prime}}$ : equivalent workstation

$A_{m_{i}}^{\prime}$ and Assigned availability and unavailability to the $\overline{A_{m_{i}}^{\prime}}$ :

\section{Data Availability}

The data used to support the findings of this study are included within the article.

\section{Conflicts of Interest}

The authors declare that they have no conflicts of interest. 


\section{Acknowledgments}

The research in this paper was supported by the National Science and Technology Major Project of China (Grant no. 2014ZX04015031), Science and Technology Development Plan Project of Jilin Province (Grant no. 20180520068JH), and Jilin Province Education Department's Thirteenth FiveYear Plan Science and Technology Project (Grant no. JJKH20180079KJ).

\section{References}

[1] Z. J. Yang, Q. B. Hao, C. Fei et al., "A comprehensive fuzzy reliability allocation method of NC machine tools based on interval analysis," Journal of Beijing University of Technology, vol. 37, no. 3, pp. 321-329, 2011.

[2] P.-C. Chang, "Reliability estimation for a stochastic production system with finite buffer storage by a simulation approach," Annals of Operations Research, vol. 277, no. 1, pp. 119-133, 2019.

[3] G. Liberopoulos, "Performance evaluation of a production line operated under an echelon buffer policy," IISE Transactions, vol. 50, no. 3, pp. 161-177, 2018.

[4] S. Weiss, J. A. Schwarz, and R. Stolletz, "The buffer allocation problem in production lines: formulations, solution methods, and instances," IISE Transactions, vol. 51, no. 5, pp. 456-485, 2019.

[5] J. Duan, A. P. Li, X. Nan et al., "Multi-state reliability modeling and analysis of reconfigurable manufacturing systems," Journal of Mechanical Engineering, vol. 47, no. 17, pp. 104-111, 2011.

[6] J. L. Li, Y. H. Jia, F. Xu, F. Chen, Z. Yang, and X. Li, “An improvement scheme for the overall line effectiveness of a production line: a case study," in Proceedings of the International Conference on System Reliability and Science (ICSRS), Singapore, April 2018.

[7] G. F. Li, Y. Li, X. G. Zang et al., "Development of a preventive maintenance strategy for an automatic production line based on group maintenance method," Applied Sciences, vol. 8, no. 10, p. 1781, 2018.

[8] G. F. Li, C. Hou, G. F. Liu, Y. Jia, C. Liu, and J. Dong, "Reliability allocation method of production line based on fuzzy comprehensive evaluation," in Proceedings of the International Conference on Materials Engineering, Manufacturing Technology and Control (ICMEMTC), Taiyuan, China, April, 2016.

[9] Y. H. Jia, Z. J. Yang, G. F. Li et al., “An efficient semi-analytical simulation for availability evaluation of discrete production lines with unreliable machines," in Proceedings of the International Conference on System Reliability and Science (ICSRS), Paris, France, November 2016.

[10] F. Chen, B. Liu, and B. B. Xu, "Research on Reliability Evaluation of Engine Cylinder Flexible Production Line Based on AHP Fuzzy Comprehensive Evaluation Method," China Mechanical Engineering Society, Changzhou, China, August 2015.

[11] F. Long, P. Zeiler, and B. Bertsche, "Modelling the flexibility of production systems in industry 4.0 for analysing their productivity and availability with high-level Petri nets," IFACPapersOnLine, vol. 50, no. 1, pp. 5680-5687, 2017.

[12] L. Görkemli and S. Kapan Ulusoy, "Fuzzy bayesian reliability and availability analysis of production systems," Computers \& Industrial Engineering, vol. 59, no. 4, pp. 690-696, 2010.
[13] S. Rebello, H. Yu, and L. Ma, "An integrated approach for system functional reliability assessment using dynamic bayesian network and hidden Markov model," Reliability Engineering \& System Safety, vol. 180, no. 12, pp. 124-135, 2018.

[14] K. Heungseob and K. Pansoo, "Reliability models for a nonrepairable system with heterogeneous components having a phase-type time-to-failure distribution," Reliability Engineering and System Safety, vol. 159, no. 3, pp. 37-46, 2017.

[15] M. K. Loganathan, G. Kumar, and O. P. Gandhi, “Availability evaluation of manufacturing systems using semi-markov model," International Journal of Computer Integrated Manufacturing, vol. 29, no. 7, pp. 720-735, 2016.

[16] X.-Y. Li, H.-Z. Huang, and Y.-F. Li, "Reliability analysis of phased mission system with non-exponential and partially repairable components," Reliability Engineering \& System Safety, vol. 175, no. 7, pp. 119-127, 2018.

[17] A. B. Hu and S. M. Meerkov, "Lean buffering in serial production lines with Bernoulli machines," Mathematical Problems in Engineering, vol. 2006, Article ID 17105, 24 pages, 2006.

[18] L. Demir, S. Tunalı, and D. T. Eliiyi, “An adaptive tabu search approach for buffer allocation problem in unreliable nonhomogenous production lines," Computers \& Operations Research, vol. 39, no. 7, pp. 1477-1486, 2012.

[19] R. Li, X. Liu, and N. Huang, "Availability allocation of networked systems using Markov model and heuristics algorithm," Mathematical Problems in Engineering, vol. 2014, Article ID 315385, 9 pages, 2014.

[20] W. X. Qian, X. W. Yin, and L. Y. Xie, "System reliability allocation based on bayesian network," Applied Mathematics \& Information Sciences, vol. 6, no. 3, pp. 681-687, 2012.

[21] M. Xiao and Y. P. Zhang, "Parameters learning of bayesian networks for multistate system with small sample," Computer Science, vol. 42, no. 4, pp. 253-257, 2015.

[22] V. Sriramdas, S. K. Chaturvedi, and H. Gargama, "Fuzzy arithmetic based reliability allocation approach during early design and development," Expert Systems with Applications, vol. 41, no. 7, pp. 3444-3449, 2014.

[23] S. G. Shu, "The manufacturing system (CIMS) with buffers and a study of the system reliability," Acta Automatica Sinica, vol. 18, no. 1, pp. 15-20, 1992.

[24] H. R. Zhou, S. H. Wang, L. Zhang et al., "Modeling of inprocess inventory in continuous production based on reliability," Machine Tool \& Hydraulics, vol. 39, no. 9, pp. 111113, 2011.

[25] M. Y. You and J. C. Zheng, "A synthetic approach to reliability allocation of electronic systems based on AGREE method and the extended proportional combination method," Electronic Product Reliability and Environmental Testing, vol. 29, no. 4, pp. 1-6, 2011. 